\title{
Probleme in Bezug auf die slowenische lexikographische Terminologie
}

\author{
Simona Štavbar, Abteilung für Germanistik, Philosophische Fakultät, \\ Universität Maribor, Slowenien (simonastavbar@gmail.com)
}

Zusammenfassung: Der Beitrag behandelt slowenische Terminologie im Bereich der lexikographischen Disziplin und die damit verbundene terminologische Problematik der lexikographischen Disziplin in Slowenien. Für diese ist ein großer Anteil an synonymen und fehlenden lexikographischen Termini charakteristisch. Im Beitrag wird gezeigt, wie und inwieweit man die fehlende slowenische lexikographische Terminologie in Anlehnung an die deutsche lexikographische Terminologie anhand der Wortbildungsverfahren einführen könnte, was zur Entwicklung der lexikographischen Theorie und Erhöhung der Qualität von lexikographischen Produkten führen würde.

Stichwörter: DEUTSCHE LEXIKOGRAPHISCHE TERMINOLOGIE, SLOWENISCHE LEXIKOGRAPHISCHE TERMINOLOGIE, SYNONYME TERMINI, FEHLENDE TERMINI, WORTBILDUNGSVERFAHREN, LEXIKOGRAPHISCHE DISZIPLIN IN SLOWENIEN

\begin{abstract}
Problems related to the Slovenian lexicographical terminology. The article discusses Slovenian terminology in the field of lexicography as well as terminological problems related to the lexicographical discipline in Slovenia. The terminology of this discipline displays a great number of synonymous lexicographical terms on the one hand, and a lack of lexicographical terms on the other hand. Based on the German lexicographical terminology, this article looks into how and to which extent the missing lexicographical terms can be formed in Slovene through different types of word-formation processes. The introduction of much needed lexicographical terms into Slovene would contribute to the formation of lexicographical theory and raise the quality of lexicographical products.
\end{abstract}

Keywords: GERMAN LEXICOGRAPHICAL TERMINOLOGY, SLOVENIAN LEXICOGRAPHICAL TERMINOLOGY, SYNONYMOUS TERMS, LACK OF TERMS, WORD FORMATION, SYNONYMOUS LEXICOGRAPHICAL TERMS, LEXICOGRAPHICAL DISCIPLINE IN SLOVENIA

\section{Zum Stand und zur Problematik der slowenischen lexikographischen} Terminologie

Im Folgenden wird die slowenische Terminologie im Bereich der lexikographischen Disziplin und die damit verbundene terminologische Problematik der lexikographischen Disziplin in Slowenien besprochen. In Anlehnung an die 
deutsche lexikographische Terminologie werden die Möglichkeiten vorgestellt, wie man die fehlende lexikographische Terminologie anhand der Wortbildungsverfahren einführen könnte und es wird darauf hingewiesen, dass man die uneinheitliche lexikographische Terminologie im Slowenischen, die sich in Synonymie niederschlägt ${ }^{1}$, vereinheitlichen sollte. Das Einführen von fehlenden lexikographischen Termini im Slowenischen würde nämlich zur Entwicklung der lexikographischen Theorie und zur Erhöhung der Qualität von lexikographischen Produkten führen. Auf die angeführte Problematik bezüglich der Fachterminologie, ihrer lexikographischen und terminographischen Erfassung macht Jesenšek aufmerksam:

Trotz der Vielzahl und Vielfalt der Fachwörterbücher, der steigenden Anzahl terminographischer Produkte und intensiver empirischer und theoriegeleiteter Forschung bleiben nicht wenige Problembereiche in der Auseinandersetzung mit den Fachsprachen und ihrer lexikographischen bzw. terminographischen Erfassung weiterhin ungeklärt. (Jesenšek 2013: 11)

Es werden zuerst die Gründe angeführt, die zur uneinheitlichen Terminologie in der slowenischen lexikographischen Disziplin beitrugen.

\section{Gründe für die uneinheitliche lexikographische Terminologie im Slo- wenischen}

Für die uneinheitliche lexikographische Terminologie, die sich in der Verwendung synonymer Termini und auch im Fehlen von lexikographischen Termini widerspiegelt, gibt es mehrere Gründe. Ihr Vorkommen ist verbunden mit der Tätigkeit unterschiedlicher terminologischer Schulen, die in der Vergangenheit bei der Begründung der Lexikographie als selbständige Disziplin ihre eigene lexikographische Terminologie formten und in die lexikographische Disziplin einführten. Als sich diese Terminologie etablierte, wurden in die Zielsprache Termini anderer Sprachen eingeführt. Im slowenischen Raum hatte besonders die Tschechische terminologische Schule ${ }^{2}$ einen relativ großen Einfluss auf die Art und den Umfang der lexikographischen Terminologie. ${ }^{3}$ Mit der späteren Etablierung unterschiedlicher Theorien bzw. theoretischer Vorschläge wurden in die Zielsprache neue Termini eingeführt. Dies führte jedoch zur uneinheitlichen Verwendung der lexikographischen Termini (z. B. der deutsche Terminus Lemma und die Varianten dieses Terminus iztočnica/slovarska iztočnica/geslo im Slowenischen). Das Angeführte ist auch das Resultat der mangelnden Vereinbarung bzw. der unzureichenden Zusammenarbeit unter den Fachleuten dieses Fachgebiets, da im institutionellen Sinne auf dem internationalen Niveau die Zusammenarbeit gut ist, auf dem nationalen Niveau diese jedoch nicht ausreichend ist. Der nächste Grund für die uneinheitliche Terminologie und für das Aufkommen von synonymen Termini ist der Einfluss anderer Disziplinen, darunter z. B. der Informatik. Hier wird die Terminologie dieser Disziplin von der lexikographischen Disziplin übernommen, wobei es wegen mangelnder 
lexikographischer Fachkenntnisse zur uneinheitlichen Verwendung der lexikographischen Termini kommt (vgl. z. B. die oft falsche Verwendung der Termini informacija und podatek im Slowenischen bzw. Informationen und Daten im Deutschen). ${ }^{4}$ Auf internationalen Konferenzen, Kongressen und Kolloquien wird überwiegend das Englische als Verständigungssprache verwendet, das Englische überwiegt auch in Fachpublikationen, wie z. B. Euralex, International Journal of Lexicography, Lexikos u. a. Für den englisch- und deutschsprachigen Raum stehen mehrere lexikographische Fachpublikationen zur Verfügung, was man für den slowenischen Raum nicht feststellen kann. Hier erscheinen Beiträge zur allgemeinen Lexikographie, Fachlexikographie und zur metasprachlichen Lexikographie überwiegend in Publikationen anderer linguistischer Disziplinen oder werden in bestimmten thematisch konzipierten Nummern der linguistischen Fachpublikationen publiziert. ${ }^{5}$ Im slowenischen Raum gibt es derzeit auch keine Quellen (Fachwörterbücher, Datenbanken u. a.), in denen lexikographische Terminologie gesammelt, entsprechend behandelt und den Benutzern zugänglich wäre. Zudem stehen auch keine Lehrwerke zu genannten Themenbereichen und auch keine Übersetzungen wichtiger lexikographischer Texte zur Verfügung, die die Beschäftigung mit lexikographischer Terminologie anregen würden. ${ }^{6}$ Aus diesem Grund ist die deutsche Metalexikographie im slowenischen Raum wenig bekannt und damit auch die Terminologie, die mit ihrer Strukturiertheit als Basis für das Einführen der fehlenden lexikographischen Terminologie und für ihre Vereinheitlichung hätte dienen können.7 Ein weiterer Grund für das Genannte liegt in der letzten Zeit bestimmt auch in den fehlenden oder unzureichenden Deutschkenntnissen (als Folge der Fremdsprachenpolitik im Ausbildungssystem); das zeigt sich an der Berücksichtigung nur der englischen Literatur zum Thema.

Im Bereich der praktischen lexikographischen Tätigkeit in Slowenien werden von mehreren nationalen Instituten und auch von Unternehmen allgemeine und fachsprachliche Wörterbücher und andere lexikographische Produkte vorbereitet (z. B. vom Inštitut za slovenski jezik Frana Ramovša, ${ }^{8}$ Institut "Jožef Stefan", ${ }^{9}$ Trojina ${ }^{10}$ oder Amebis $\left.{ }^{11}\right)$, im Bereich der theoretischen Lexikographie lassen sich jedoch Schwierigkeiten bezüglich der Verwendung der lexikographischen Terminologie beobachten. In Fachpublikationen und in Wörterbuchaußentexten gibt es oft Fälle von synonymen lexikographischen Termini, die im Folgenden an Belegen dargestellt werden.

\section{Zur synonymen Verwendung der Termini in der slowenischen lexiko- graphischen Disziplin und die fehlende Terminologie}

In slowenischen linguistischen Fachpublikationen und in Wörterbuchaußentexten wird der lexikographische Terminus iztočnica [Lemma] oft synonym verwendet, u. z. gibt es die Varianten iztočnica, geslo, geselska iztočnica und slovarska iztočnica. In der Fachliteratur ist die Form iztočnica etabliert, die auch im SP $(2001)^{12}$ und im SSKJ2 (2014) ${ }^{13}$ belegt ist sowie in Wörterbuchaußentexten und 
in Fachartikeln. Im SSKJ (1994) ${ }^{14}$ ist der Terminus geslo belegt (vgl. Uvod [Einführung]); iztočnica ist dort mit der Häufigkeitsangabe redko [selten] markiert. Im SP (2001) sind beide Termini belegt. Das Gleiche gilt auch für das SSKJ2 (2014). Der Terminus iztočnica hat sich, den Quellen zufolge, erst mit der Zeit etabliert. In Verbindung mit dem Terminus geslo ist im SP (2001) auch der Terminus geselski članek belegt, der im SSKJ2 (2014) als slovarski sestavek belegt ist und an anderen Stellen noch als geselski sestavek [Wörterbuchartikel]. In Fachartikeln werden in Verbindung mit iztočnica die Termini varianta iztočnice und iztočnična varianta angeführt. Im SSKJ2 (2014) ist der Terminus različica (iztočnice) belegt, was das slowenische Äquivalent zum entlehnten Terminus varianta (iztočnice) ist. Im Deutschen handelt es sich hierbei um Zusammensetzung, u. z. Lemmavariante. Als Einwort- bzw. Mehrwortterminus ist ustreznik bzw. tujejezični ustreznik/tuji ustreznik und ustreznica bzw. tujejezična ustreznica belegt. Das erste Beispiel bezieht sich auf Termini und das zweite auf Lexeme. Im Deutschen verwendet man in beiden Fällen den Terminus Äquivalent. Synonym kommen auch terminološki kvalifikator und terminološki označevalnik [Fachgebietsangabe] vor, wobei dieser Terminus auch deskriptiv als označevalnik s področja strokovne rabe belegt ist. An diesem und auch am Beispiel von varianta (iztočnice)/ različica (iztočnice) lässt sich beobachten, dass die entlehnte und die slowenische Form oft parallel vorkommen. Als Synonyme sind Termini besednovrstni podatek und besednovrstni kvalifikator belegt, was im Deutschen Wortartangabe ist. Das slowenische Äquivalent des deutschen Terminus Belegbeispielangabe ist im SSKJ2 (2014) als Ein- bzw. Mehrwortterminus zgled[i] und zgledi iz dejanske rabe angegeben. Das Angeführte deutet auf einen relativ großen Anteil an synonymen Termini und auf den Bedarf nach Vereinheitlichung bzw. Standardisierung der lexikographischen Terminologie im Slowenischen.

Neben der Vereinheitlichung besteht zudem der Bedarf nach den lexikographischen Termini, die es im Slowenischen nicht gibt. Das Einführen der fehlenden lexikographischen Termini würde nämlich eine höhere Qualität von lexikographischen Beschreibungen sichern. Erarbeiten und anbieten müsste man z. B. Benennungen für die Arten der Wörterbücher, u. z. fachliches Sprachwörterbuch (*jezikovni strokovni slovar), ${ }^{15}$ fachliches Sachwörterbuch $\left({ }^{*}\right.$ stvarni strokovni slovar), monoinformatives Wörterbuch (*enoizborni slovar), Aussprachewörterbuch (*izgovorni slovar), polyinformatives Wörterbuch (*večizborni slovar), Sprachstadienwörterbuch ( ${ }^{*}$ slovar jezikovnega obdobja/slovar jezikovnih obdobij) u.a. In Verbindung mit dem Lemma z. B. Lemmakandidat ( ${ }^{* k a n d i d a t ~ z a ~ i z t o c ̌ n i c o), ~ L e m-~}$ mazeichen (iztočnični znak?) und Lemmazeichengestaltangabe (oblika iztočničnega znaka?). Zudem gibt es keine Benennungen für die Bestandteile des Wörterbuchartikels und für Strukturen, z.B. glattalphabetische Makrostruktur ( ${ }^{*}$ tekočeabecedna makrostruktura), nischenalphabetische Makrostruktur ( ${ }^{*}$ besedotvorno tekočeabecedna makrostruktura), nestalphabetische Makrostruktur ( ${ }^{*}$ prekinjena tekočeabecedna makrostruktura), einfache Mikrostruktur ( ${ }^{*}$ enostavna mikrostruktura),

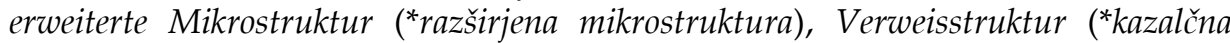
struktura), typographischer Strukturanzeiger ( ${ }^{*}$ tipografski strukturni kazalnik), nicht- 
typographischer Strukturanzeiger ( ${ }^{*}$ netipografski strukturni kazalnik) und viele andere lexikographische Termini.

\section{Eigenschaften der Termini}

Wie anfangs angegeben wurde, führt zur Erweiterung des lexikographischen Fachwortschatzes das Verfahren der Wortbildung. Um den Fachwortschatz im Bereich der Lexikographie im Slowenischen zu erweitern, werden zudem Lexeme aus anderen Sprachen entlehnt, wobei hier die Gebersprache das Deutsche ist. ${ }^{16} \mathrm{Im}$ Folgenden wird kontrastiv vorgegangen, u. z. werden die lexikographischen Termini im Slowenischen in Anlehnung an das Deutsche angeführt und angeboten, da das Deutsche die Gebersprache für die slowenischen Termini ist und die slowenische lexikographische Terminologie in Anlehnung an das deutsche terminologische System und unter Berücksichtigung der deutschen lexikographischen Theorie festgelegt werden soll. ${ }^{17}$

\subsection{Wortbildung}

Nach Fleischer und Barz (2012: 18) handelt es sich bei der Wortbildung um „die Produktion von Lexemen auf der Grundlage und mithilfe vorhandenen Sprachmaterials." Das Sprachmaterial wird immer morphosyntaktisch verändert (Donalies 2002: 19). Wortbildung umfasst die Verfahren, die man für die Bildung von Lexemen zur Verfügung hat und auch den Bestand an wortgebildeten Lexemen sowie deren kommunikative Fähigkeiten (Fleischer und Barz 2012: 1). Die am häufigsten verwendeten Verfahren der Wortbildung sind auch im Bereich der Bildung lexikographischer Termini im Deutschen und Slowenischen - die Komposition und die Derivation. Zudem gibt es noch die Konversion und Kurzwortbildung, die in der Fachlexikographie mit Deutsch sehr oft zur Bildung von Sachnamen eingesetzt wird.

Wortbildung hat einen wichtigen Anteil an der Erweiterung des lexikographischen Fachwortschatzes. Im Folgenden wird somit näher auf die Verfahren der Komposition, Derivation, Konversion, Kurzwortbildung und Entlehnung sowie Lehnwortbildung eingegangen.

\subsubsection{Komposition}

Komposita sind komplexe Lexeme, die sich aus zwei wortfähigen unmittelbaren Konstituenten zusammensetzen. Ihrer Abfolge nach werden die beiden unmittelbaren Konstituenten entweder als Erstglied oder als Zweitglied im Lexem unterschieden (Fleischer und Barz 2012: 84). Die Bildung von zusammengesetzten Benennungen ist im Deutschen sehr produktiv (Donalies 2002: 53). Das gilt auch für den Bereich der lexikographischen Terminologie und für die Terminologie aus anderen, benachbarten Fachgebieten, wo Komposita und 
Mehrworttermini besonders zahlreich bzw. dominierend sind. Es gibt folgende Möglichkeiten zur Bildung von Komposita im Deutschen:

a) Substantiv + Substantiv

Fachlexikographie, Typologiegraph, Wörterbuchfunktion, Wörterbuchtyp, Rezeptionswörterbuch, Abkürzungswörterbuch, Synonymenwörterbuch, Antonymenwörterbuch, Archaismenwörterbuch, Neologismenwörterbuch, Sachwörterbuch, Sprachwörterbuch, Lemmazeichen, Lemmavariante, Formkommentar, Wörterbuchartikel, Lemmaliste, Datenbank, Synonymenangabe, Phrasemangabe, Lemmakandidat

b) Verbstamm + Substantiv Verweisstruktur, Zugriffsstruktur, Planungsphase

c) Adjektiv + Substantiv

Primärquelle, Sekundärquelle, Spezialwörterbuch, Kurzwort, Leitelement, Fremdwörterbuch

d) Adverb + Substantiv

Außentext, Vorspann, Nachspann

e) Numerale + Substantiv

Letztbeleg, Allbuch, Erstbeleg

Im Vergleich zum Deutschen lässt sich im Slowenischen eine wesentlich geringere Wortbildungsproduktivität im Bereich der Komposition feststellen. Die aus dem Deutschen entlehnten Termini werden im Slowenischen in der Mehrheit der Fälle als attributive Wortverbindungen realisiert, u.z. gibt es jeweils ein Substantiv als Bezugswort, das links oder rechts attribuiert ist, wobei die Fälle mit Linksattribuierung überwiegen:

Substantiv + Linksattribut

(1)

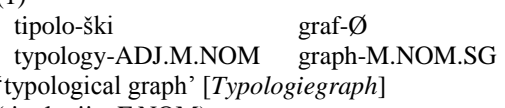
'typological graph'
(tipologija, F.NOM)

(3)

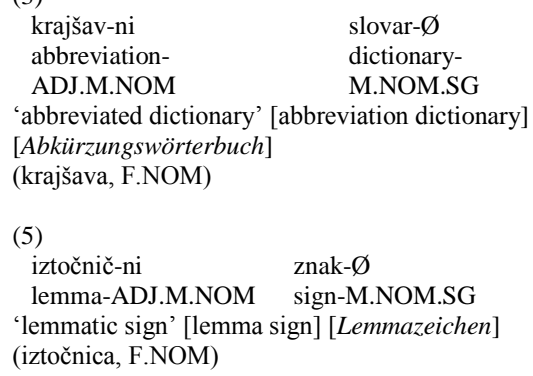

(2)

$\begin{array}{ll}\text { recepcij-ski } & \text { slovar- } \varnothing \\ \text { reception- } & \text { dictionary- } \\ \text { ADJ.M.NOM } & \text { M.NOM.SG }\end{array}$

ADJ.M.NOM M.NOM.SG

'receptive dictionary' [reception dictionary]

[Rezeptionswörterbuch] (recepcija, F.NOM)

(4)

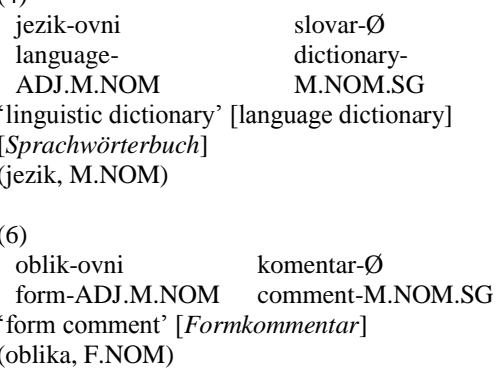


(7)

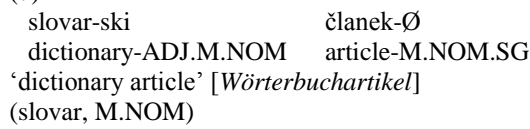

(9)

načrtov-(a)lna faz-a

plan-ADJ.F.NOM phase-F.NOM.SG

'planning phase' [Planungsphase]

(načrtovati, INF)

(11)

sekundar-ni vir- $\varnothing$

secondary-M.NOM source-M.NOM.SG

'secondary source' [Sekundärquelle]

(sekundaren, ADJ; cf. German: sekundär)

(13)

poseb-ni $\quad$ slovar- $\varnothing$

'separately'[special] ${ }^{1}$ dictionary-

M.NOM.SG

'special dictionary' [Spezialwörterbuch]

(posebej, ADV)

*ADV=adverb; $1=$ shift of meaning

\section{Substantiv + Rechtsattribut}

(1)

$\begin{array}{ll}\text { funkcij-a } & \text { slovarj-a } \\ \text { function-F.NOM.SG } & \text { dictionary-M.GEN.SG } \\ \text { function of a dictionary' } & \text { [Wörterbuchfunktion] }\end{array}$

(3)

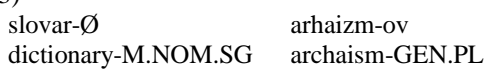

(5)

$\begin{array}{ll}\text { različic-a } & \text { iztočnic-e } \\ \text { variant-F.NOM.SG } & \text { lemma-F.GEN.SG } \\ \text { 'variant of a lemma' [Lemmavariante] }\end{array}$

(7)

struktur-a
structure-F.NOM.SG dostopanj-a
structure of access' [Zugriffsstruktur]

(8)

$\begin{array}{ll}\text { kazalč-na } & \text { struktur-a } \\ \text { cross-reference- } & \text { structure- } \\ \text { ADJ.F NOM } & \text { F NOM SG }\end{array}$

'cross-reference structure' [Verweisstruktur] (kazalec, M.NOM)

(10)

primar-ni $\quad$ vir- $\varnothing$
primary-M.NOM $\quad$ source-M.NOM.SG
primary source' [Primärquelle]

'primary source' [Primärquelle]

(primaren, ADJ; cf. German: primär)

(12)

zun-anje besedil-o

out-ADJ.N.NOM text-N.NOM.SG

'outer text' $[$ Außentext $]$

(zunaj, $\mathrm{ADV}^{*}$ )

(14)

uvod-ni del- $\varnothing$

introduction-ADJ.M.NOM part-M.NOM.SG

'introductory part' [Vorspann]

(uvod, M.NOM)
(2)

$\begin{array}{ll}\text { vrst-a } & \text { slovarj-a } \\ \text { type-F.NOM.SG } & \text { dictionary-M.GEN.SG } \\ \text { type of a dictionary' } & {[\text { Wörterbuchtyp] }}\end{array}$

(4)

slovar- $\varnothing$ neologizm-ov

dictionary-M.NOM.SG. neologism-GEN.PL

'dictionary of neologisms' [Neologismenwörterbuch]

(6)

seznam- $\varnothing \quad$ iztočnic- $\varnothing$
list-M.NOM.SG
list of lemmata' [Lemmaliste]

'list of lemmata' [Lemmaliste]

(8)

slovar- $\varnothing \quad$ tujk- $\varnothing$

dictionary-M.NOM.SG foreign word-GEN.PL

'dictionary of foreign words' [Fremdwörterbuch]

In einigen Fällen stehen im Slowenischen beide Möglichkeiten zur Verfügung, oft als paralleles Vorkommen von entlehntem (sog. Internationalismen) und einheimischem Sprachmaterial: ${ }^{18}$

(1) Synonymenwörterbuch $\rightarrow$ slovar sinonimov/sopomenski slovar/sinonimni slovar,

(2) Antonymenwörterbuch $\rightarrow$ slovar antonimov/protipomenski slovar/antonimni slovar 


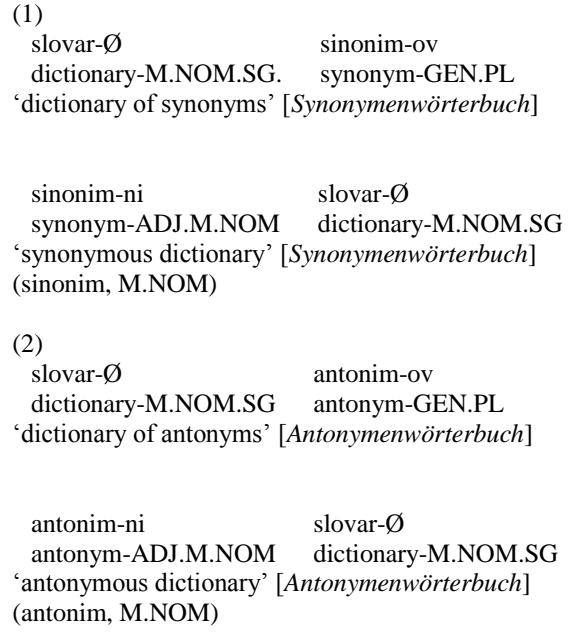

Einige Beispiele deutscher lexikographischer Termini werden im Slowenischen als präpositionale Wortverbindungen realisiert:

(1) Synonymenangabe $\rightarrow$ podatek o sinonimu, (2) Phrasemangabe $\rightarrow$ podatek o frazemu, (3) Lemmakandidat $\rightarrow$ kandidat za iztočnico

(1)

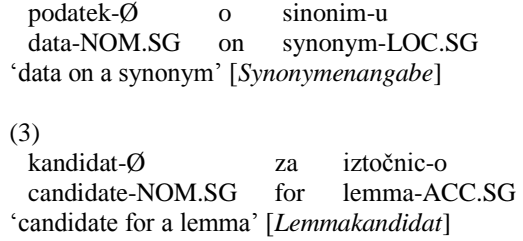

(2)

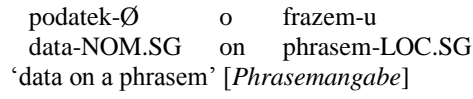

Aus dem bislang Angeführten ist ersichtlich, dass Zusammensetzungen im Slowenischen zwar vorhanden sind, sie spielen jedoch eine untergeordnete Rolle. Zudem gibt es vereinzelt Fälle von deutschen Zusammensetzungen, die im Slowenischen als nichtattribuierte Einworttermini belegt sind, z. B. (1) Nestanordnung $\rightarrow$ gnezdenje, (2) Nachspann $\rightarrow$ dodatek

(1)

gnezdenj-e

nesting-N.NOM.SG

'nesting' ${ }^{[}$Nestanordnung $]$

1 =substantive; 2 =shift of meaning
(2)

dodatek- $\varnothing$

appendix [back matter] ${ }^{2}$-M.NOM.SG

'appendix' [back matter] [Nachspann]

Bei längeren Zusammensetzungen in den Fachsprachen lässt sich beobachten, dass auch hier wie in der Allgemeinsprache das Verfahren der Reduktion einsetzt (Poethe 2000: 204). Mittels Kürzung lassen sich ökonomischere Benennungen erzeugen. Lexikographische terminologische Einheiten in Form von Zusammensetzungen werden in einigen Fällen bei der ersten Anführung im 
Text in ihrer Grundform angeführt, bei jeder weiteren Erwähnung jedoch in der Kurzform:

Lesartenettiketierung $\rightarrow$ Ettiketierung, Kurzettiketierung; Lesartendisambiguierung $\rightarrow$ Disambiguierung; Sprachnorm $\rightarrow$ Norm, Registerhaupteintrag $\rightarrow$ Haupteintrag, Artikelnester $\rightarrow$ Nester, Artikelnischen $\rightarrow$ Nischen, Wörterbuchartikel $\rightarrow$ Artikel, Mikrostrukturanzeiger $\rightarrow$ Strukturanzeiger, Artikelmikrostruktur $\rightarrow$ Mikrostruktur

Solche Grundformen mit den dazugehörigen Kurzformen lassen sich für das Slowenische, mit einigen Ausnahmen (z. B. (1) slovarska iztočnica $\rightarrow$ iztočnica [Lemma], (2) zgled [i] iz dejanske rabe $\rightarrow$ zgled[i]) [Belegbeispielangabe( $n)$ ], nicht belegen. Der Grund dafür liegt in der wenig ausgearbeiteten lexikographischen Terminologie im Slowenischen, die eine geringe Strukturiertheit des lexikographischen terminologischen Systems und der Relationen zwischen den Begriffen aufweist.

(1)

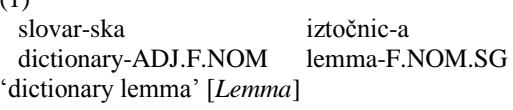

Daneben hat das Deutsche grundsätzlich die Möglichkeit, relativ lange und komplexe Einwortbenennungen zu bilden. Diese sind in der lexikographischen Terminologie des Deutschen recht zahlreich vertreten und stellen in Fällen von terminologischen Lücken in anderen Sprachen ein kaum lösbares Übersetzungsproblem dar (Butina-Koller et al. 2010: XXV-XXX), besonders, weil man sie oft nur in Form von festen Wortverbindungen übersetzen kann, was zugleich eine Abweichung vom Formaspekt des Terminus bedeutet. Besonders problematisch ist das in Fällen, wo es im Deutschen zu Bildungen mit hoher Gliederanzahl kommt: Verweisadressangabe, Belegbeispielangabe, Wörterbuchbenutzungstest, Wörterbuchbenutzungsforschung, Datendistributionsstruktur, Fachwörterbuchartikel, Sprachstadienwörterbuch, Artikelstrukturschema, Lemmazeichengestaltangabe, Lemmakandidatenliste

Für solche Zusammensetzungen im Deutschen gilt, dass sie im Slowenischen in der Regel durch attributive oder präpositionale Wortverbindungen wiedergegeben werden, wobei man im Slowenischen besonders eine hohe Anzahl von mehrgliedrigen attributiven Verbindungen beobachten kann:

(1) Wörterbuchbenutzungstest $\rightarrow$ test rabe slovarja, (2) Wörterbuchbenutzungsforschung $\rightarrow$ raziskovanje rabe slovarja, (3) Datendistributionsstruktur $\rightarrow$ struktura porazdelitve podatkov, (4) Fachwörterbuchartikel $\rightarrow$ slovarski članek strokovnega slovarja, (5) Sprachstadienwörterbuch $\rightarrow$ slovar jezikovnega obdobja, (6) Artikelstruk- 
turschema $\rightarrow$ shema strukture slovarskega članka, (7) Lemmakandidatenliste $\rightarrow$ seznam kandidatov za iztočnico

(1) $\begin{array}{lll}\text { test- } \varnothing & \text { rab-e } & \text { slovarj-a } \\ \text { test- } & \text { use- } & \text { dictionary- } \\ \text { M.NOM.SG } & \text { F.GEN.SG } & \text { M.GEN.SG } \\ \text { 'test of dictionary } & \text { use' [Wörterbuchbenutzungstest] }\end{array}$

(3)

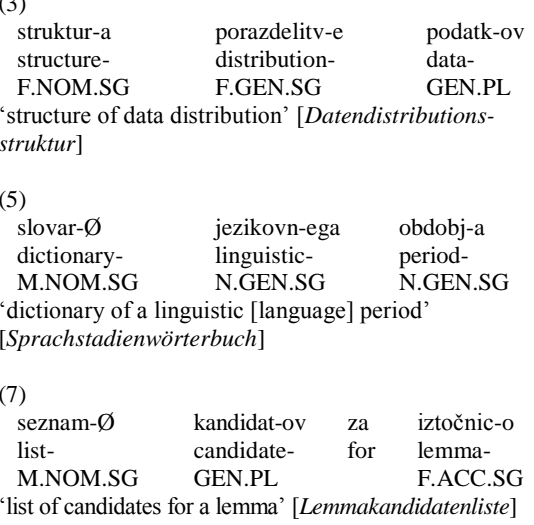

(2) raziskovanj-e rab-e slovarj-a research- use- dictionaryN.NOM.SG F.GEN.SG M.GEN.SG 'research of dictionary use' [Wörterbuchbenutzungsforschung]

(4)

slovar-ski članek- $\varnothing \quad$ strokovn-ega slovarj-a dictionary- article- $\quad$ specialized- dictionaryADJ.M.NOM M.NOM.SG M.GEN.SG M.GEN.SG 'dictionary article of a specialized dictionary' [Fachwörterbuchartikel]

(6) shem-a struktur-e slovarsk-ega člank-a F.NOM.SG F.GEN.SG M.GEN.SG M. GEN.SG 'scheme of a structure of a dictionary article' [Artikelstrukturschema]

In Fällen von Mehrwortbenennungen gibt es im Deutschen zahlreiche Beispiele terminologischer Bildungen, bei denen sich die Struktur des Terminus aus einem oder mehreren attributiven Adjektiven vor einem substantivischen Kompositum oder einer Ableitung zusammensetzt oder die Struktur Genitivbzw. Präpositionalattribut-Argumente in Nachstellung enthält. Die deutsche lexikographische Terminologie ist nämlich durch ein erhöhtes Erscheinen von komplexen Lexemen charakterisiert. Solche komplexe Strukturen haben einen differenzierenden und spezifizierenden Charakter. Beispiele solcher Bildungen sind in der lexikographischen Terminologie zahlreich: textbezogene Wörterbuchfunktion, wissensbezogene Wörterbuchfunktion, abstrakte hierarchische linke Kernstruktur, konkrete hierarchische rechte Kernstruktur, basaler Wörterbuchartikel, typographischer Strukturanzeiger, nicht-typographischer Strukturanzeiger, lexikographische Datenerhebung

Wie in vielen bislang angeführten Beispielen, in denen kein slowenischer Terminus nachgewiesen werden konnte, werden slowenische lexikographische Termini in solchen Fällen durch eine wörtliche Übersetzung geschaffen. Da sich das Wortbildungssystem im Slowenischen von dem Deutschen unterscheidet, wirken viele slowenische Termini nicht natürlich, was besonders an den unteren Mehrwortbildungen in Form von längeren Wortketten ersichtlich ist:

(1) textbezogene Wörterbuchfunktion $\rightarrow$ slovarska funkcija $v$ zvezi z besedilom, (2) wissensbezogene Wörterbuchfunktion $\rightarrow$ slovarska funkcija v zvezi z znanjem, (3) abstrakte hierarchische linke Kernstruktur $\rightarrow$ abstraktna hierarhična leva jedrna struktura, (4) konkrete hierarchische rechte Kernstruktur $\rightarrow$ konkretna hierarhična desna jedrna struk- 
tura, (5) basaler Wörterbuchartikel $\rightarrow$ temeljni slovarski članek, (6) typographischer Strukturanzeiger $\rightarrow$ tipografski strukturni kazalnik, (7) lexikographische Datenerhebung $\rightarrow$ slovaropisno pridobivanje podatkov

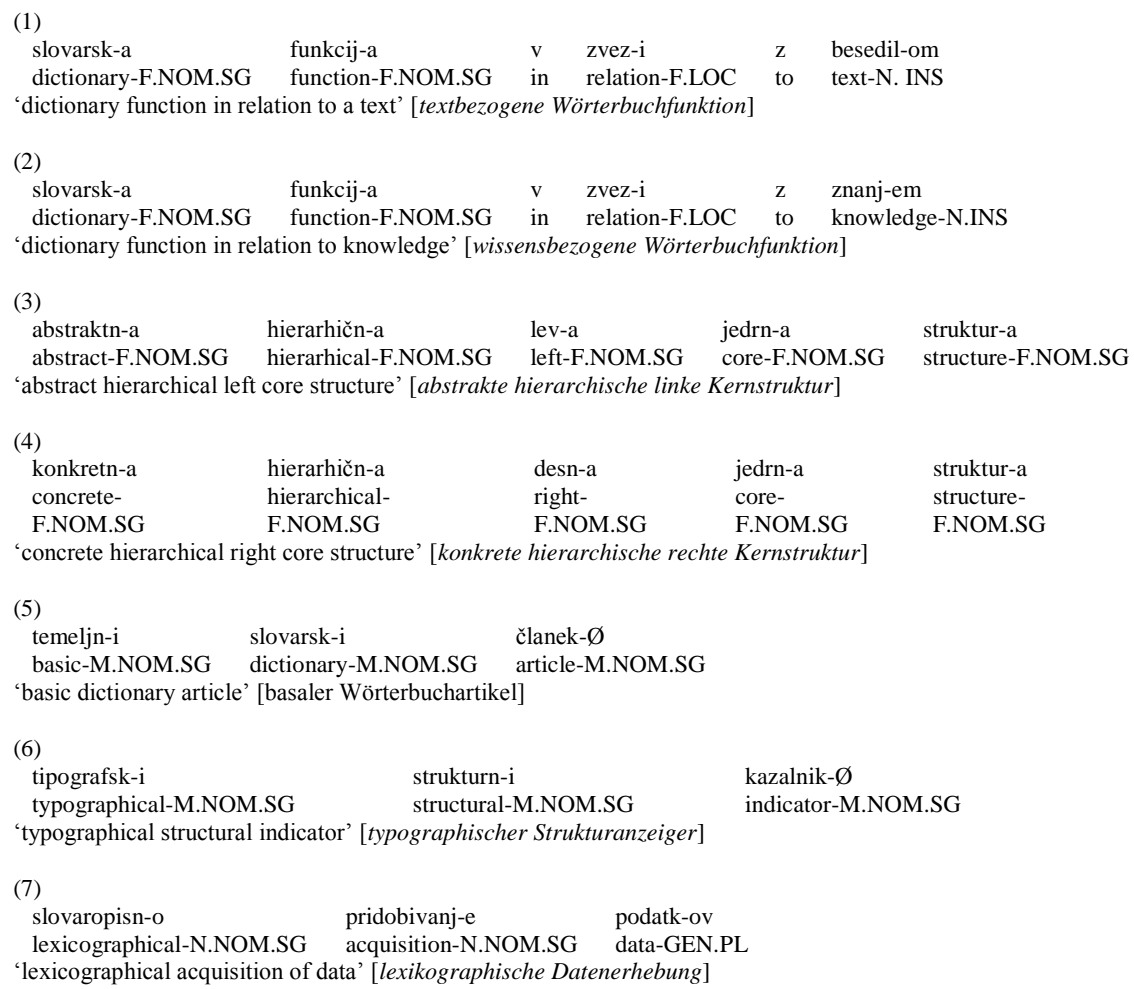

\subsubsection{Derivation}

Bei der Wortableitung bzw. Derivation handelt es sich um die Verbindung einer Derivationsbasis (Wortstamm, Konfix oder Syntagma) mit einem Derivationsaffix (Präfix, Suffix oder Zirkumfix) (vgl. Fleischer und Barz 2012: 86). Die Suffixderivation betrifft vor allem Substantive und Adjektive, die Präfixderivation und Konversion sind für Verben charakteristisch (ebd.).

Im Folgenden werden die Wortbildungsprodukte der Derivation - die Suffixderivation, die Präfixderivation und die Zirkumfixderivation - an Beispielen der Lexeme aus dem Bereich der Lexikographie angeführt:

a1) Suffixderivation bei Substantiven

Charakteristisch hierfür sind folgende Suffixe: -e (Liste, Experte), -ei/-erei (Datei), -el (Artikel), -er (Benutzer, Nutzer), -heit/-keit/-igkeit (Häufigkeit, Prüfbarkeit), -nis (Verzeichnis), -(i)s (Basis), -ung (Markierung, Adressierung). Solche Belege werden 
im Slowenischen entweder als Simplex, als Ableitung oder als Zusammensetzung realisiert. Simplex im Slowenischen ist: (1) seznam [Liste; Verzeichnis]; Belege für die Suffixableitung sind: (2) strokovnjak [Experte], (3) uporabnik [Nutzer, Benutzer], (4) sestavek [Artikel], (5) pogostost [Häufigkeit], (6) preverliivost [Prüfbarkeit], (7) osnova [Basis], (8) označevanje [Markierung], (9) naslavljanje [Adressierung]; Zusammensetzung: (10) datoteka [Datei].

(1)

seznam- $\varnothing$

list-NOM.SG

'list' [Liste; Verzeichnis]

(3)

uporab-nik

use-NOM.SG (suffix: -nik)

'user' [Nutzer, Benutzer]

(uporaba, $\mathrm{S}^{*}$ )

(5)

\section{pogost-ost}

frequent-NOM.SG (suffix: -ost)

'frequency' [Häufigkeit]

(pogost, ADJ)

(7)

osnov-a

basic-NOM.SG (suffix: -a)

'basis' [Basis]

(9)

naslavlj-anje

address-NOM.SG (suffix: -anje)

'address' [Adressierung]

(nasloviti, V)

$* \mathrm{~V}=$ verb, $\mathrm{S}=$ substantive
(2)

strokovn-jak

expert-NOM.SG (suffix: -jak)

'expert' [Experte]

(strokoven, ADJ)

(4)

sestav-ek

compile-NOM.SG (suffix: -ek)

'compilation' [article] [Artikel]

(sestaviti, $\mathrm{V}^{*}$ )

(6)

preverljiv-ost

verifiable-NOM.SG (suffix: -ost)

'verification' [Prüfbarkeit]

(preverljiv, ADV)

(8)

označ-evanje

label-NOM.SG (suffix: -evanje)

'label' [Markierung]

(označiti, V)

(10)

datotek-a

file-NOM.SG (stem: dat-; interfixal formant:

'file' [Datei]

(stem: dat-; interfixal fort
-o-; stem: -(biblio)teka

$($ podatkovna $+($ biblio $)$ teka $))$

\section{a2) Suffixderivation bei Adjektiven}

Man findet folgende Suffixe: -bar (prüfbar), -er (inner, basaler), -ich (fachlich) -isch (lexikographisch, typographisch), -los (erfolglos). Im Slowenischen werden die obigen Beispiele als Präfix- bzw. Suffixableitung realisiert. Präfixableitung: (1) neuspešen [erfolglos]; Belege für die Suffixableitung: (2) preverljiv [prüfbar], (3) notranji [inner], (4) temeljni [bazaler], (5) strokoven [fachlich], (6) leksikografski [lexikographisch], (7) tipografski [typographisch].

(1)

ne-uspeš-en

NEG-success-

ADJ.M.NOM

'unsuccessful' [erfolglos]

(uspeh, M.NOM)
(2) prever-(1)jiv test-ADJ.M.NOM (suffix: -(l)jiv) 'testable' [prüfbar] (preveriti, V) 
(3)

notr-anji
inside-ADJ.M.NOM (suffix: -anji)
'inner' [inner]
(noter, ADV)

(5)

strok-oven

special field-ADJ.M.NOM (suffix: -oven)

'special field"' [fachlich]

(stroka, F.NOM.)

(7)

tipograf-ski

typography-ADJ.M.NOM (suffix: -ski)

'typographical' [typographisch]

(tipografija, F.NOM.)

$1=$ adj. only before a noun: special field dictionary
(4)

temelj-ni

basis-ADJ.M.NOM (suffix: -ni)

'basic' [bazaler]

(6)

leksikograf-ski

lexicography-ADJ.M.NOM (suffix: -ski)

'lexicographical' [lexikographisch

a3) Suffixderivation bei Verben

Für die Suffixderivation bei Verben gibt es nur wenige produktive Suffixe, u. z. -el(n)/-l(n) (vermitteln), -er(n)/-r(n) (verändern), -ier(en)/-isier(en)/-efizier(en) (standardisieren, lemmatisieren, sortieren, publizieren). Die angegebenen Belege werden im Slowenischen als Präfixableitung realisiert: (1) posredovati [vermitteln], (2) spremeniti [verändern]; Belege für die Suffixableitung sind: (3) standardizirati [standardisieren], (4) lematizirati [lemmatisieren], (5) urejati [sortieren], (6) objaviti [publizieren].

(1)

po-sred-ovat

PREP*-middle- (prefix: po-, verbal suffix:

INF -ova-, verbal formant: -ti)

'to mediate' [to communicate $]^{1}$ [vermitteln]

(3)

standard-

izirati

standard-INF (verbal suffix: -izira-, verbal

formant: -ti)

'to standardize' [standardisieren]

(5)

urej-ati

sorted-INF (verbal suffix: -a-, verbal

'to sort' [sortieren]

formant: -ti)

*PREP=preposition; $1=$ shift of meaning
(2)

s-premen-iti

PREP-change- (prefix: s-, verbal suffix: -i-,

INF verbal formant: -ti)

'to change' [verändern]

(4)

lemat-izirati

lemma-INF (verbal suffix: -izira-, verbal

formant: -ti)

'to lemmatize'[lemmatisieren]

(6)

objav-iti

publication-INF (verbal suffix: -i-, verbal

‘to publish' [publizieren

b1) Präfixderivation bei Substantiven

Es lassen sich folgende Präfixe beobachten: haupt- (Hauptlemma), ur- (Urbeleg, Urliste). Im Slowenischen wird der erste Beleg als attributive Wortverbindung realisiert: glavna iztočnica [Hauptlemma], bei den zwei darauffolgenden Belegen handelt es sich um Fälle von Bildungen mit dem Präfix -pra und um Beispiele der Wortbildungsart "sestavljanje bzw. sestava“19 (Präfigierung): (1) praprimer [Urbeleg], (2) praseznam [Urliste]. 
(1)

praprimer- $\varnothing$

original.example-NOM.SG (prefix: pra-)

'original example' [Urbeleg]
(2) praseznam- $\varnothing$ original.list-NOM.SG (prefix: pra-) 'original list' [Urliste]

b2) Präfixderivation bei Adjektiven

Die Anzahl der Präfixe ist gering (Fleischer und Barz 2012: 351): un- (unabhängig, unmittelbar). Im Slowenischen handelt es sich um Fälle von "sestavljanje bzw. sestava": (1) neodvisen [unabhängig], (2) neposreden [unmittelbar].

(1)

ne-odvis-en

NEG-depend-ADJ.M.NOM (prefix: ne-)

'independent' [unabhängig]

$1=$ shift of meaning
(2)

ne-posred-en

NEG-in.the.middle-ADJ.M.NOM (prefix: ne-) 'not in the middle' [immediate, direct] ${ }^{1}$ [unmittelbar

b3) Präfixderivation bei Verben

Nach Fleischer und Barz (2012: 380) gehört die Präfixderivation zu den in der verbalen Wortbildung am häufigsten genutzten Wortbildungsarten. Verbale Präfixderivate sind untrennbar. Hier gibt es folgende Präfixe: be- (benutzen, bearbeiten), ver- (verweisen, umverteilen), zer- (zergliedern), über- (überprüfen), um- (umschreiben), unter- (unterstützen). Im Slowenischen handelt es sich bei den folgenden Fällen um Präfixderivation: (1) uporabiti [benutzen], (2) obdelati [bearbeiten], (3) usmerjati [verweisen], (4) prerazporediti [umverteilen], (5) razčleniti [zergliedern], (6) preveriti [überprüfen], (7) opisati [umschreiben], (8) podpreti [unterstützen].

(1)

$$
\begin{array}{ll}
\text { u-porab-iti } & \\
\text { PREP-use-INF } & \text { (prefix: u-, } \\
& \text { verbal suffix: -i-, verbal } \\
& \text { formant: -ti) }
\end{array}
$$

'to use' [benutzen]

(3)

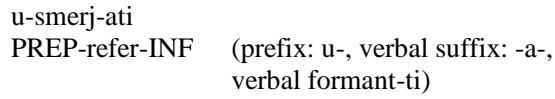

(5)

raz-člen-iti

ADV-segment- (prefix: raz-, verbal suffix

INF -i-, verbal formant: $-\mathrm{ti}$ )

'to segment' [zergliedern]

(7)

o-pis-ati

$\mathrm{X}^{*}$-describe- (prefix: o-, verbal suffix: -a-, INF verbal formant: -ti)

'to describe' [umschreiben]

$* \mathrm{X}=$ no specification possible;
(2)

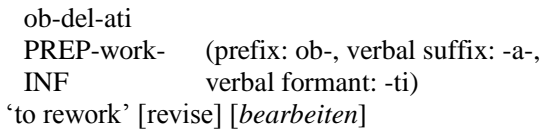

(4)

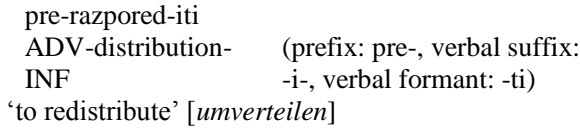

(6)

pre-ver-iti

ADV-verify- (prefix: pre-, verbal suffix: -i-, INF verbal formant: -ti)

'to verify' [überprüfen]

(8) pod-pr-eti

ADV- (prefix: pod-, verbal suffix: -e-, 'support'- verbal formant: -ti); ADV pod INF meaning spodaj, i.e. under; -preti 'to support' [unterstützen]

\section{meaning push, press}


c1) Zirkumfixderivation bei Adjektiven ${ }^{20}$

Hier ist die Derivation nicht auf die Kombinationen mit ge- beschränkt, z. B. inter- (interfachlich). Das Beispiel hier ist im Slowenischen ein Beleg für die Wortbildungsart "sestavljanje bzw. sestava“: (1) medstrokoven [interfachlich].

(1)

med-strok-oven

PREP-discipline-ADJ.M.NOM (prefix: med-)

'interdisciplinary' [interfachlich]

c2) Zirkumfixderivation bei Verben

Die Derivation mit Zirkumfixen be-/ver- ... -ig(en) und ver- ...-ier(en) ist schwach ausgebaut. Hierbei entstehen untrennbare Verben (Fleischer und Barz 2012: 433), z. B. beabsichtigen. Im Slowenischen handelt es sich um Präfixderivation: (1) nameravati [beabsichtigen].

(1)

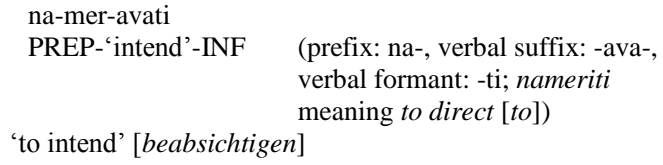

\subsubsection{Konversion}

Bei der Konversion handelt es sich um den Übergang von Lexemen einer Wortklasse in die andere (vgl. Fleischer und Barz 2012: 87). Man unterscheidet zwei Arten von Konversion, u.z. die morphologische und die syntaktische Konversion. Im Falle der morphologischen Konversion „stehen zwei phonologisch gleiche Stämme in einer Motivationsbeziehung zueinander" (ebd.: 88). Beispiele der morphologischen Konversion im Bereich der lexikographischen Lexik sind z. B. verweis(en) $\rightarrow$ Verweis, beleg $(e n) \rightarrow$ Beleg u.a. ${ }^{21}$ In Fällen mit parallelem Vorkommen von Verb und abstraktem Substantiv ist aus der synchronen Perspektive jedoch schwierig zu bestimmen, in welche Richtung die Konversion verläuft (vgl. ebd.).

Bildungen mit $A b-, A n-, Z u$ - sind Konversionen von trennbar zusammengesetzten Verben: zugreifen $\rightarrow$ Zugriff, angeben $\rightarrow$ Angabe, abstehen $\rightarrow$ Abstand.

Um syntaktische Konversion handelt es sich, wenn das Konvertat ein Flexionselement seiner Basis behält, z. B. das grammatische Morphem -en in Fällen des substantivierten Infinitivs (vgl. ebd.): nachschlagen $\rightarrow$ Nachschlagen, adressieren $\rightarrow$ Adressieren, wissen $\rightarrow$ Wissen, nutzen $\rightarrow$ Nutzen, lesen $\rightarrow$ Lesen u. a. Aus dem Angeführten ist ersichtlich, dass es sich um gemeinsprachliche Lexeme handelt, die auch in der Lexikographie verwendet werden.

Im Vergleich zum Deutschen gibt es hier jedoch durch die Sprachstruktur bedingte Unterschiede. Typisch für das Slowenische ist, dass Verben gewöhnlich als Basis für Substantivableitungen dienen, u.z. mit oder ohne Wortbil- 
dungsaffixe. In dieser Hinsicht gibt es Fälle der Suffixableitung mit Nullsuffix: (1) dostopati $\rightarrow$ dostop, (2) nasloviti $\rightarrow$ naslov, (3) odmakniti $\rightarrow$ odmik, oder Fälle mit Suffixableitung: (4) rabiti $\rightarrow$ raba, (5) poiskati $\rightarrow$ iskanje.

(1)

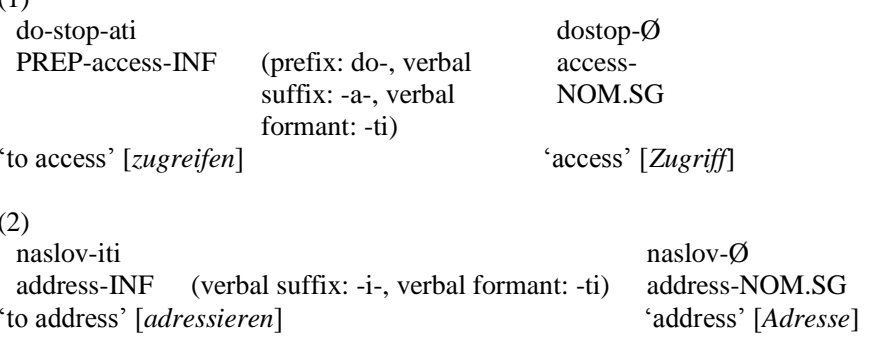

(3) od-mak-niti

PREP-move-INF (prefix: od-, verbal suffix: -ni-, verbal formant: -ti)

odmik- $\varnothing$

'to remove' [entfernen]

removal-NOM.SG

'removal'[Entfernung]

(4)

rab-iti rab-a

use-INF (verbal suffix: -i-, verbal formant: -ti) use-NOM.SG (suffix: -a)

'to use' [verwenden] 'usage' [Verwendung]

(5)

$\begin{array}{lll}\text { po-isk-ati } & \text { isk-anje } \\ \text { PREP-consult-INF } & \text { (prefix: po-, verbal suffix: -a-, verbal } & \begin{array}{l}\text { consult-NOM.SG } \\ \text { formant: -ti) }\end{array} \\ \text { 'to consult'[nachschlagen }] & \text { 'consultation' [Nachschlagen }]\end{array}$

\subsubsection{Kurzwortbildung}

Neben den behandelten Wortbildungsarten Komposition und Derivation werden lexikographische Fachausdrücke auch mittels Kürzung gebildet. Kurzwörter sind Produkte unterschiedlicher Kürzungsvorgänge, bei denen die längere Vollform reduziert wird, sie haben Wortcharakter und werden als Benennungen verwendet (Fleischer und Barz 1995: 218; 2012: 91). Dem Vorteil der Benutzung von Kurzwörtern, der vor allem in der Sprachökonomie liegt, kann man als Nachteil insbesondere einen Mangel an Verständlichkeit und Präzision gegenüber der längeren Form hervorheben. Kurzwörter sind nämlich häufig mehrdeutig, wenn sie sich auf mehrere Fachgebiete und auf mehrere Sprachen beziehen (Arntz, Picht und Mayer 2009: 120). Somit erweisen sie sich als problematisch, da man mit dem gleichen Kurzwort etwas Unterschiedliches benennt. ${ }^{22}$ Kurzwörter haben manchmal einen differenzierenden Charakter, da ähnliche Benennungen mittels Kurzwörter leichter unterschieden werden. In Verbindung mit der Wortbildung im Bereich der Lexikographie lässt sich die Tendenz feststellen, dass Kurzwörter neben den sprachökonomischen auch andere Funktionen haben. Interessanterweise fungieren sie im Deutschen auch 
als Titel, also als Sachnamen für lexikographische Nachschlagewerke, z. B. DWDS (Digitales Wörterbuch der deutschen Sprache), WLWF (Wörterbuch zur Lexikographie und Wörterbuchforschung), OWID (Online-Wortschatz-Informationssystem Deutsch) u. a. Dies gilt nicht nur für das Deutsche, sondern auch für das Slowenische, z. B. SSKJ (Slovar slovenskega knjižnega jezika) [Wörterbuch der slowenischen Standardsprache], SMeS (Slovenski medicinski elektronski slovar) [Slowenisches elektronisches Wörterbuch der Medizin] oder Evroterm (Terminološka zbirka izrazov Evropske unije) [Terminologische Sammlung von Ausdrücken der Europäischen Union], GETS (Geodetski tezaver in slovar) [Thesaurus und Wörterbuch der Geodäsie], wobei der Anteil dieser im Slowenischen im Vergleich zum Deutschen deutlich geringer ist. ${ }^{23}$

Hier sei besonders auf die folgenden Kurzformtypen verwiesen:

a) Abkürzungen, die entstehen, wenn eine Benennung nicht in ihrer vollen Form ausgeschrieben wird. Dieser Typ von Kurzformen ist in der lexikographischen Terminologie, besonders in Wiegands Fachtexten häufig vorhanden und wird dort bei grafischen Darstellungen hauptsächlich aus praktischen Gründen eingesetzt, vgl. z. B. Lematis. = Lemmatisierung, Homographentr. = Homographentrennung, exh. $=$ exhaustiv, initial.$=$ initialalphabetisch, final.$=$ final alphabetisch (1998: 205-206). Solche Abkürzungen werden in der Fachliteratur auch Gebrauchsabkürzungen bezeichnet (vgl. Fleischer und Barz 1995: 218). Für das Slowenische sind sie nicht charakteristisch.

b) Initialwörter (Akronyme), bei denen es sich um die aus dem Anfangsbuchstaben mehrerer Wörter entstandene neue Ausdrucksform handelt (vgl. Bußmann 1990: 42). Wenn eine solche Form wie ein Wort ausgesprochen wird, liegt eine (1) „Sprechkürzung“24 vor und wenn nicht, dann handelt es sich um Fälle von (2) „Buchstabierkürzung“ (Arntz, Picht und Mayer 2009: 121). In der lexikographischen und computerlinguistischen Terminologie findet man Beispiele von beidem:

1. Beispiele für "Sprechkürzung" im Deutschen:

KWIC $\rightarrow$ key word in context, OWID $\rightarrow$ Online-Wortschatz-Informationssystem Deutsch, OBELEX $\rightarrow$ Online-Bibliografie zur elektronischen Lexikographie, COSMAS

$\rightarrow$ Corpus Search, Management and Analysis System, OWL $\rightarrow$ Web Ontology Language

1a Beispiel für "Sprechkürzung" im Slowenischen:

GETS $\rightarrow$ Geodetski tezaver in slovar [Thesaurus und Wörterbuch der Geodäsie], SMeS

$\rightarrow$ Slovenski medicinski slovar [Slowenisches elektronisches Wörterbuch der Medizin]

2. Beispiele für "Buchstabierkürzung“ im Deutschen:

DWDS $\rightarrow$ Digitales Wörterbuch der deutschen Sprache, WLWF $\rightarrow$ Wörterbuch zur Lexikographie und Wörterbuchforschung, DTD $\rightarrow$ Document Type Definition, XML 
$\rightarrow$ eXtensible Markup Language, WDG $\rightarrow$ Wörterbuch der deutschen Gegenwartssprache, MLR $\rightarrow$ multifunktionale lexikalische Ressource

2a Beispiele für „Buchstabierkürzung im Slowenischen: SSKJ $\rightarrow$ Slovar slovenskega knjižnega jezika [Wörterbuch der slowenischen Standardsprache], SSSJ $\rightarrow$ Sprotni slovar slovenskega jezika [Growing Dictionary of the Slovenian Language], 25 SNB $\rightarrow$ Slovar novejšega besedja [Wörterbuch des neueren Wortschatzes]

c) Silbenkurzwörter, die aus dem Anfang des Wortes und weiteren Silben einer Ausgangsbenennung bestehen, eignen sich besonders für die Bildung eines einprägsamen und problemlos aussprechbaren Wortes (Arntz, Picht und Mayer 2009: 121). Diese Art der Wortkürzung ist im Deutschen oft vorhanden (vgl. z. B. elexiko $\rightarrow$ elektronisches, lexikalisch-lexikographisches korpusbasiertes Informationssystem oder dereko $\rightarrow$ das deutsche Referenzkorpus). In diesem und in einigen oben angeführten Beispielen zeigt sich bei der Kurzwortbildung die Tendenz, Namen für Wörterbücher und andere Informationssysteme zu bilden. Somit ist die Kurzwortbildung hier auf Sachnamenbildung ausgerichtet.

\subsection{Entlehnung und Lehnwortbildung}

Nach Fleischer und Barz (2012: 102) entsteht der Fremdwortschatz im Deutschen "durch Entlehnung "fertiger" Wörter und durch Wortbildung mit exogenen Elementen innerhalb des Deutschen". Unter Entlehnung versteht man die unveränderte bzw. weitgehend unveränderte Übernahme eines Lexems aus einer anderen Sprache - auf sprachliches Material wird also außerhalb der gleichen Sprache gegriffen (vgl. Donalies 2002: 16).

Bei der Lehnwortbildung greift man auf die 'modernen' Gebersprachen (z. B. das Englische) oder auf die 'klassischen' Sprachen zurück (das Griechische oder das Lateinische). ${ }^{26}$ Die griechischen und lateinischen Wortstämme als exogene Wortbildungseinheiten gelangen ins Deutsche oft auch über andere Sprachen (z. B. über das Englische) (vgl. Fleischer und Barz 2012: 104).

Fleischer und Barz (2012: 102) unterscheiden bei der Lehnwortbildung zwei Gruppen von motivierten Wortbildungen. Zur ersten Gruppe gehören die Bildungen mit exogenen Einheiten und zur zweiten Gruppe gehören Kombinationen aus exogenen und indigenen Einheiten. Im Bereich der lexikographischen Terminologie gehören zur ersten Gruppe Lexeme wie computerextern, Kontentvalidierung, Hyperlink, Reliabilität u. a. Für die zweite Gruppe lässt sich bezüglich der Lehnwortbildung beobachten, dass sich ein exogenes Erstglied oft mit einem indigenen Zweitglied (oft sind das Substantive) verbindet. Dies lässt sich an folgenden Beispielen der lexikographischen Termini beobachten: Mediostruktur, Mikrostruktur, Mikrostrukturanzeiger, Makrostruktur. Diese Teilung auf zwei Gruppen gilt auch für das Slowenische, wobei sich die deutschen und die slowenischen Belege bezüglich der exogenen und indigenen Einheiten oft 
nicht überlappen. Zur ersten Gruppe gehören z. B. (1) mediostruktura [Mediostruktur], (2) mikrostruktura [Mikrostruktur], (3) makrostruktura [Makrostruktur] und zur zweiten Gruppe z. B. (4) hiperpovezava [Hyperlink].

(1)

mediostruktur-a

middle.structure-F.NOM.SG

'middle structure' [Mediostruktur]

(3)

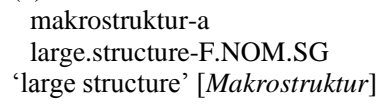

(2) mikrostruktur-a
very small.structure-F.NOM.SG
very small structure' [Mikrostruktur

(4) hiperpovezav-a

beyond the usual size.link-F.NOM.SG 'link beyond the usual size' [Hyperlink]

Auch in der Fremdwortbildung unterscheidet man die Wortbildungsarten der Komposition, Derivation, Konversion und Kurzwortbildung. Hier sei nur kurz auf die Möglichkeiten der Komposition und Derivation im Bereich der lexikographischen Terminologie eingegangen. Bei der Komposition unterscheidet man zwei Gruppen, u.z. 1. Komposita aus zwei wortfähigen Konstituenten, von denen jeweils eine oder zwei exogen sind und 2. Komposita aus zwei Konfixen oder aus einem Konfix und einer wortfähigen Konstituente (Fleischer und Barz 2012: 111).27

1. Komposita mit einer bzw. zwei exogenen Konstituenten im Deutschen: Portallemmaliste, Computerunterstützung, Konsultationspraxis

Im Slowenischen werden die angegebenen Termini unterschiedlich realisiert. Zum einen ist die Gebersprache für die slowenische Terminologie das Deutsche (praksa dostopanja [Konsultationspraxis], seznam iztočnic portala [Portallemmaliste]), zum anderen das Englische (računalniška podpora [computer support]). Auch bezüglich der Wortbildungsverfahren lassen sich Unterschiede nachweisen, u. z. werden diese Termini im Slowenischen als attributive Wortverbindungen realisiert - mit dem exogenen Sprachmaterial: (1) seznam iztočnic portala, (2) praksa dostopanja oder ohne: (3) računalniška podpora.

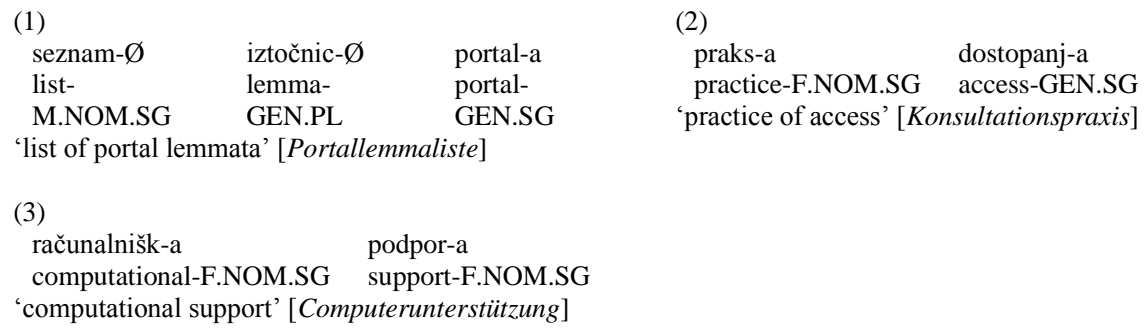

2. Konfixkomposita aus einem bzw. aus zwei Konfixen und einer wortfähigen Konstituente im Deutschen:

Mikroarchitektur, Metalexikographie, Metatext, Kontext, Mediostruktur, Mikrostruktur, Makrostruktur 
Im Slowenischen bestehen die Zusammensetzungen entweder aus einer oder aus mehreren exogenen unmittelbaren Konstituenten mikro-/meta-/medio-/makro-: (1) mikrozgradba [Mikrostruktur], (2) metabesedilo [Metatext], (3) mediostruktura [Mediostruktur], (4) mikrostruktura [Mikrostruktur], (5) maktrostruktura [Makrostruktur]. Zudem gibt es Zusammensetzungen mit der indigenen unmittelbaren Konstituente sam- in (6) samodejen [automatisch] und sestavljanke mit der indigenen Konstituente so-: (7) sobesedilo [Kontext].

(1)

mikrozgradb-a

very small.structure-F.NOM.SG

'very small structure' [Mikrostruktur]

(3)

mediostruktur-a

middle.structure-F.NOM.SG

'middle structure' [Mediostruktur]

(5)

makrostruktur-a

large.structure-F.NOM.SG

'large structure' [Makrostruktur]

(7)

sobesedil-o

surrounding.text-N.NOM.SG

'context' [Kontext $]$
(2)

metabesedil-o

at a higher level.text-N.NOM.SG

'text at a higher level' [Metatext]

(4)

mikrostruktur-a

very small.structure-F.NOM.SG

'very small structure' [Mikrostruktur]

(6)

samodej-en

self-acting-ADJ.M.NOM

'self-acting' [automatisch]

Bezüglich der Derivation gibt es die folgenden Möglichkeiten der Kombination von exogenen und indigenen Wortbildungseinheiten, wobei hier die Grenze zwischen der Fremdwortbildung und indigener Wortbildung fließend ist (vgl. Fleischer und Barz 2012: 112):

1. exogene Basis und indigenes Suffix:

-ung (Digitalisierung), -heit (Differenziertheit), -schaft (Autorschaft), -al (digital), -e (solide), -el(l) (flexibel, maschinell), -ion (Annotation, Kollokation) -isch (typographisch, symmetrisch), -ieren (definieren, strukturieren, analysieren).

Im Slowenischen gibt es die exogene oder die indigene Basis mit indigenem bzw. exogenem Suffix. Exogene Basis und exogenes bzw. indigenes Suffix weisen folgende Termini auf: (1) diferenciranost [Differenziertheit], (2) avtorstvo [Autorschaft], (3) digitalen [digital], (4) fleksibilen [flexibel], (5) anotacija [Annotation], (6) kolokacija [Kollokation], (7) tipografski [typographisch], (8) simetričen [symmetrisch], (9) definirati [definieren], (10) strukturirati [strukturieren], (11) analizirati [analysieren] (12) digitalizacija [Digitalisierung]; indigene Basis und indigenes Suffix weisen folgende Belege auf: (13) zanesljiv [solide], (14) strojen [maschinell].

(1)

diferenciran-ost

differentiated-F.NOM.SG

'differentiation' [Differenziertheit]
(2)

avtor-stvo

author-N.NOM.SG

‘authorship' [Autorschaft] 
(3)

digital-en

digitize-ADJ.M.NOM

'digital' [digital]

(5)

anot-acija

annotate-NOM.SG

'annotation' [Annotation]

(7)

tipograf-ski

typography-ADJ.M.NOM

'typographic' [typographisch]

(9)

defin-irati

definition-INF

'to define' [definieren]

(11)

analiz-irati

analysis-INF

'to analyse' [analysieren]

(13)

zanes-ljiv

rely.on-ADJ.M.NOM

'reliable' [solide]
(4)

fleksibil-en

fexibility-ADJM.NOM

'flexible' [flexibel]

(6)

kolok-acija

collocate-NOM.SG

'collocation' [Kollokation]

(8)

simetr-ičen

symmetry-ADJ.M.NOM

'symmetric' [symmetrisch]

(10)

struktur-irati

structure-INF

'to structure' [strukturieren]

(12)

digitaliz-acija

digitize-NOM.SG

'digitalization' [Digitalisierung]

(14)

stroj-en

machine-ADJ.M.NOM

'machine-assisted' [maschinell]

2. indigene Basis und exogenes Suffix:

-ieren (alphabetisieren)

Im Slowenischen ist sowohl die Basis als auch das Suffix exogen: alfabetirati. Dieser Terminus wird jedoch im Slowenischen meist als Wortverbindung (1) abecedna razporeditev [alphabetische Anordnung] realisiert.

(1) $\begin{array}{lc}\text { abeced-na } & \text { razporeditev- } \varnothing \\ \text { alphabet-ADJ.F.NOM } & \text { arrangement-F.NOM.SG } \\ \text { 'alphabetical arrangement' } & \text { [alphabetische Anordnung] }\end{array}$

3. exogene Basis und indigenes Präfix: un- (unstrukturiert, unmarkiert)

Bei den folgenden Beispielen handelt es sich im Slowenischen um sestavljanje bzw. sestava mit dem indigenen Präfix ne- und der exogenen bzw. indigenen Basis: (1) nestrukturiran [unstrukturiert], (2) neoznačen [unmarkiert].

(1)

NEG-structure-ADJ.M.NOM (prefix: ne-)
'unstructured' [unstrukturiert]
(2)

ne-označ-en

NEG-mark-ADJ.M.NOM (prefix: ne-) 'unmarked' [unmarkiert] 
4. indigene Basis und exogenes Präfix:

in- (indirekt), sub- (Subkommentar)

Im Slowenischen geht es um Beispiele von sestavljenje oz. sestava, u.z. mit der indigenen Basis und dem indigenen Präfix: (1) neposreden [indirekt], (2) podkomentar [Subkommentar].

(1)

ne-posred-en

NEG-direct-ADJ.M.NOM (prefix: ne-)

'indirect' [indirekt]
(2)

pod-komentar- $\varnothing$

PREP-comment-M.NOM.SG (prefix: pod-)

'sub-comment' [Subkommentar]

Die Mehrheit der neuen Termini bringt heute die technologische Entwicklung mit sich, also ein neuer Begriff führt zum neuen Terminus. Es passiert zudem oft, dass man mit der Übernahme einer technischen Neuentwicklung auch die Benennung aus der betreffenden Sprache mit übernimmt. Besonders in den letzten Jahren könnte man eine Menge der englischen Fachwörter auf einigen sich schnell entwickelnden Fachgebieten beobachten, die ins Deutsche übernommen wurden. Entlehnung aus modernen Sprachen erweist sich als die produktivste Art der Entlehnung (vgl. Vintar 2008: 51). Die schnelle Entwicklung von Informationswissenschaften sorgt dafür, dass Termini aus dem englischen Sprachraum übernommen werden, was sich konkret am Anteil der englischen Termini im Fachbereich der Computerlexikographie widerspiegelt (Link, Marker, Parser, Semantic Web, Tag, Tagging 28 und usage notes, Extensible Markup Language, guide word, Hyperlink u. a.). Im Slowenischen werden zu vielen englischen Entsprechungen eigene Termini gebildet, z. B. link $\rightarrow$ povezava, marker $\rightarrow$ označevalnik, parser $\rightarrow$ razčlenjevalnik, semantic web $\rightarrow$ semantični splet, tag $\rightarrow$ značka, tagging $\rightarrow$ označevanje, usage notes $\rightarrow$ oznake, Extensible Markup Language $\rightarrow$ razširljivi označevalni jezik, hyperlink $\rightarrow$ nadpovezava. Cabré (1999: 90) führt an, dass das Entlehnen von Termini oft unnötig ist, da die Nehmersprachen selbst über das Potenzial verfügen, eigene Termini zu bilden.

Im Gegensatz zur Lehnwortbildung überträgt man bei der Lehnübersetzung die einzelnen Wortelemente in die Zielsprache ohne die innere Struktur der Benennung zu verändern (z. B. Computer Aided Translation $\rightarrow$ maschinengestützte Übersetzung [računalniško podprto prevajanje], conceptual graph $\rightarrow$ konzeptueller Graph [konceptualni graf], artificial intelligence $\rightarrow$ künstliche Intelligenz [umetna inteligenca], extensible markup language $\rightarrow$ erweiterbare Auszeichnungssprache [razširljivi označevalni jezik]. Solche Lehnübersetzungen bedient man sich im Slowenischen besonders dann, wenn in Anlehnung an das deutsche lexikographische System komplexe Termini im Slowenischen gebildet werden sollen, z. B. lexikographische Datenauswertung $\rightarrow$ (1) slovaropisna analiza podatkov, Datendistributionsstruktur $\rightarrow(2)$ struktura porazdelitve podatkov, hierarchische vollständig erweiterte Mikrostruktur $\rightarrow$ (3) hierarhična popolnoma razširjena mikrostruktura, konkrete hierarchische linke Kernstruktur $\rightarrow(4)$ konkretna hierarhična leva jedrna struktura u. a. 


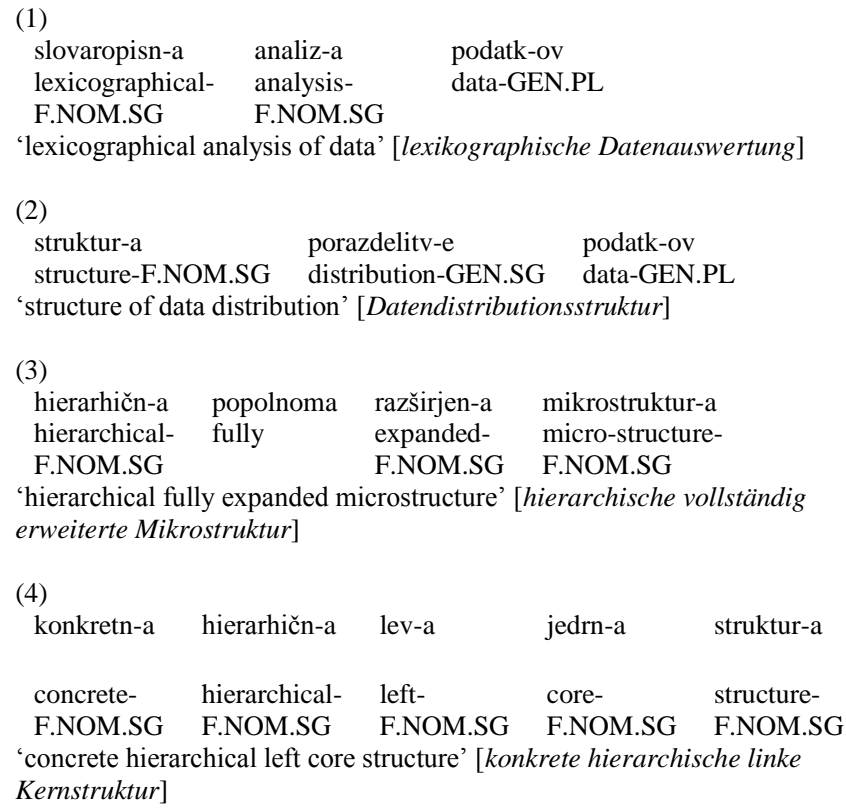

In einigen Fällen wird im Deutschen nur ein Teil der Benennung unverändert übernommen - gewöhnlich ist das die erste Konstituente - und der andere Teil wird übersetzt (Log-File-Analyse, Log-File-Protokoll, Bottom-up-Lexikographie, Offline-Wörterbuch, Think-Aloud-Protokoll, Markup-Sprache). Das Gleiche lässt sich auch für die aus dem Deutschen entlehnte lexikographische Terminologie im Slowenischen beobachten: glattalphabetische Makrostruktur $\rightarrow$ tekočeabecedna makrostruktura, nicht-typographischer Strukturanzeiger $\rightarrow$ netipografski strukturni kazalnik, computergestützte Lexikographie $\rightarrow$ računalniško podprta leksikografija u.a. $\mathrm{Zu}$ erwähnen ist hier auch der Fall der sogenannten internen Entlehnung, d. h. der Übergang bzw. die Übernahme einer Benennung in eine andere Fachsprache zur Bezeichnung eines verwandten Begriffs. Dies führt zur Mehrdeutigkeit bzw. Polysemie (vgl. z. B. den deutschen Terminus Stichwort und den slowenischen Terminus geslo).

\section{Zusammenfassende Bemerkungen}

Im Hinblick auf die lexikographische Terminologie lassen sich folgende allgemeine Charakteristika beobachten. Durch die Verfahren der Komposition und Derivation wird die lexikographische Terminologie durch Wortschatzerweiterung ausgebaut, Spezifizierung führt zur Erhöhung der Genauigkeit in der lexikographischen Terminologie. Durch das Vorgehen der Kürzung erfolgt die Steigerung der Wirtschaftlichkeit. Konversion resultiert durch den Verzicht des Agens in der Erhöhung der Anonymität. Auf der Basis der Wortbildungsverfahren lassen sich konkret folgende Charakteristika anhand des kontrastiven 
Vergleichs der deutschen und der slowenischen lexikographischen Terminologie feststellen: Deutsche Komposita werden im Slowenischen als attributive bzw. als präpositionale Wortverbindungen realisiert. Zusammensetzungen sind im Slowenischen zwar vorhanden, sie haben jedoch eine untergeordnete Rolle. Daneben hat das Deutsche grundsätzlich die Möglichkeit, relativ lange und komplexe Einwortbenennungen zu bilden, wobei diese ins Slowenische meist übersetzt werden, was zugleich eine Abweichung vom Formaspekt des Terminus bedeutet. Bezüglich der Derivation lässt sich beobachten, dass deutsche Derivate im Slowenischen unterschiedlich realisiert werden. Im Vergleich zum Deutschen gibt es bezüglich der Konversion durch die Sprachstruktur bedingte Unterschiede. Typisch für das Slowenische ist, dass Verben gewöhnlich als Basis für Substantivableitungen dienen, u. z. mit oder ohne Wortbildungsaffixe. Kurzwortbildung ist in beiden Sprachen realisiert. Bei der Lehnwortbildung lässt sich zudem beobachten, dass sich die deutschen und die slowenischen Belege bezüglich der exogenen und indigenen Einheiten oft nicht überlappen. Bei der Lehnwortbildung ist neben dem Deutschen auch das Englische (besonders in der Computerlexikographie) die Gebersprache für die slowenische Terminologie. Im Slowenischen werden zu vielen englischen Entsprechungen eigene Termini gebildet. Mithilfe der Lehnübersetzung werden, wie oben angegeben, in Anlehnung an das deutsche lexikographische System komplexe Termini im Slowenischen gebildet. Aus dem Angeführten ist ersichtlich, dass man nur für die deutschen Komposita und Kurzwortbildungen Muster für das Überführen der deutschen lexikographischen Terminologie ins Slowenische aufstellen kann, für die anderen Verfahren gilt dies nicht, was auch die oben besprochenen Belege zeigen.

Die behandelten lexikographischen Termini im Deutschen, die Termini bzw. Lexeme, die auf dem Fachgebiet der Lexikographie verwendet werden und die entsprechenden Äquivalente im Slowenischen sind in der folgenden Tabelle angeführt: ${ }^{29}$

\begin{tabular}{|l|l|}
\hline $\begin{array}{l}\text { Lexikographische Termini im Deutschen und } \\
\text { die Termini/Lexeme, die auf dem Fachgebiet } \\
\text { der Lexikographie verwendet werden } \\
\text { (alphabetisch angeordnet) }\end{array}$ & $\begin{array}{l}\text { Slowenische Äquivalente zu den lexikographischen } \\
\text { Termini im Deutschen und zu den, auf dem Fach- } \\
\text { gebiet der Lexikographie verwendeten Termini/ } \\
\text { Lexemen }\end{array}$ \\
\hline Abkürzungswörterbuch & krajšavni slovar \\
\hline abstrakte hierarchische linke Kernstruktur & abstraktna hierarhična leva jedrna struktura \\
\hline adressieren & nasloviti \\
\hline Adresse & naslov \\
\hline Adressierung & naslavljanje \\
\hline alphabetische Anordnung & abecedna razporeditev \\
\hline analysieren & analizirati \\
\hline Annotation & anotacija \\
\hline
\end{tabular}




\begin{tabular}{|c|c|}
\hline Antonymenwörterbuch & slovar antonimov \\
\hline Äquivalentangabe & podatek o ustreznici \\
\hline Archaismenwörterbuch & slovar arhaizmov \\
\hline Artikelstrukturschema & shema strukture slovarskega članka \\
\hline Außentext & zunanje besedilo \\
\hline Aussprachewörterbuch & izgovorni slovar \\
\hline automatisch & samodejen \\
\hline Autorschaft & avtorstvo \\
\hline basaler Wörterbuchartikel & temeljni slovarski članek \\
\hline Basis & osnova \\
\hline bazaler & temeljni \\
\hline beabsichtigen & nameravati \\
\hline bearbeiten & obdelati \\
\hline Belegbeispielangabe & zgled iz dejanske rabe \\
\hline benutzen & uporabiti \\
\hline Benutzer & uporabnik \\
\hline computergestützte Lexikographie & računalniško podprta leksikografija \\
\hline Computerunterstützung & računalniška podpora \\
\hline Datei & datoteka \\
\hline Datenbank & podatkovna baza \\
\hline Datendistributionsstruktur & struktura porazdelitve podatkov \\
\hline definieren & definirati \\
\hline Differenziertheit & diferenciranost \\
\hline digital & digitalen \\
\hline Digitalisierung & digitalizacija \\
\hline einfache Mikrostruktur & enostavna mikrostruktura \\
\hline entfernen & odmakniti \\
\hline Entfernung & odmik \\
\hline erfolglos & neuspešen \\
\hline erweiterbare Auszeichnungssprache & razširljivi označevalni jezik \\
\hline erweiterte Mikrostruktur & razširjena mikrostruktura \\
\hline Experte & strokovnjak \\
\hline Fachgebietsangabe & podatek o stroki \\
\hline Fachlexikographie & strokovna leksikografija \\
\hline
\end{tabular}




\begin{tabular}{|c|c|}
\hline fachlich & strokoven \\
\hline fachliches Sachwörterbuch & stvarni strokovni slovar \\
\hline fachliches Sprachwörterbuch & jezikovni strokovni slovar \\
\hline Fachwörterbuchartikel & slovarski članek strokovnega slovarja \\
\hline flexibel & fleksibilen \\
\hline Formkommentar & oblikovni komentar \\
\hline Fremdwörterbuch & slovar tujk \\
\hline glattalphabetische Makrostruktur & tekočeabecedna makrostruktura \\
\hline Häufigkeit & pogostost \\
\hline Hauptlemma & glavna iztočnica \\
\hline hierarchisch vollständig erweiterte Mikrostruktur & hierarhično popolnoma razširjena mikrostruktura \\
\hline Hyperlink & hiperpovezava \\
\hline indirekt & neposreden \\
\hline inner & notranji \\
\hline interfachlich & medstrokoven \\
\hline Kollokation & kolokacija \\
\hline konkrete hierarchische linke Kernstruktur & konkretna hierarhična leva jedrna struktura \\
\hline konkrete hierarchische rechte Kernstruktur & konkretna hierarhična desna jedrna struktura \\
\hline Konsultationspraxis & praksa dostopanja \\
\hline Kontext & sobesedilo \\
\hline künstliche Intelligenz & umetna inteligenca \\
\hline Leitelement & vodilni element \\
\hline Lemma & iztočnica \\
\hline Lemmakandidat & kandidat za iztočnico \\
\hline Lemmakandidatenliste & seznam kandidatov za iztočnico \\
\hline Lemmaliste & seznam iztočnic \\
\hline lemmatisieren & lematizirati \\
\hline Lemmavariante & različica iztočnice \\
\hline Lemmazeichen & iztočnični znak \\
\hline Lemmazeichengestaltangabe & oblika iztočničnega znaka \\
\hline lexikographisch & leksikografski \\
\hline lexikographische Datenauswertung & slovaropisna analiza podatkov \\
\hline lexikographische Datenerhebung & slovaropisno pridobivanje podatkov \\
\hline Liste & seznam \\
\hline
\end{tabular}




\begin{tabular}{|c|c|}
\hline Makrostruktur & makrostruktura \\
\hline Markierung & označevanje \\
\hline maschinell & strojen \\
\hline maschinengestützte Übersetzung & računalniško podprto prevajanje \\
\hline Mediostruktur & mediostruktura \\
\hline Metatext & metabesedilo \\
\hline Mikrostruktur & mikrostruktura \\
\hline monoinformatives Wörterbuch & enoizborni slovar \\
\hline nachschlagen & poiskati \\
\hline Nachschlagen & iskanje \\
\hline Nachspann & dodatek \\
\hline Neologismenwörterbuch & slovar neologizmov \\
\hline nestalphabetische Makrostruktur & prekinjena tekočeabecedna makrostruktura \\
\hline Nestanordnung & gnezdenje \\
\hline nicht-typographischer Strukturanzeiger & netipografski strukturni kazalnik \\
\hline nischenalphabetische Makrostruktur & besedotvorno tekočeabecedna makrostruktura \\
\hline Nutzer & uporabnik \\
\hline Phrasemangabe & podatek o frazemu \\
\hline Planungsphase & načrtovalna faza \\
\hline polyinformatives Wörterbuch & večizborni slovar \\
\hline Portallemmaliste & seznam iztočnic portala \\
\hline Primärquelle & primarni vir \\
\hline prüfbar & preverljiv \\
\hline Prüfbarkeit & preverljivost \\
\hline publizieren & objaviti \\
\hline Rezeptionswörterbuch & recepcijski slovar \\
\hline Sachwörterbuch & stvarni slovar \\
\hline Sekundärquelle & sekundarni vir \\
\hline solide & zanesljiv \\
\hline sortieren & urejati \\
\hline Spezialwörterbuch & posebni slovar \\
\hline Sprachstadienwörterbuch & slovar jezikovnega obdobja \\
\hline Sprachwörterbuch & jezikovni slovar \\
\hline standardisieren & standardizirati \\
\hline
\end{tabular}


268 Simona Štavbar

\begin{tabular}{|c|c|}
\hline Stichwort & geslo \\
\hline strukturieren & strukturirati \\
\hline Subkommentar & podkomentar \\
\hline symmetrisch & simetričen \\
\hline Synonymenangabe & podatek o sinonimu \\
\hline Synonymenwörterbuch & slovar sinonimov \\
\hline textbezogene Wörterbuchfunktion & slovarska funkcija v zvezi z besedilom \\
\hline typographisch & tipografski \\
\hline typographischer Strukturanzeiger & tipografski strukturni kazalnik \\
\hline Typologiegraph & tipološki graf \\
\hline überprüfen & preveriti \\
\hline umschreiben & opisati \\
\hline umverteilen & prerazporediti \\
\hline unabhängig & neodvisen \\
\hline unmarkiert & neoznačen \\
\hline unmittelbar & neposreden \\
\hline unstrukturiert & nestrukturiran \\
\hline unterstützen & podpreti \\
\hline Urbeleg & praprimer \\
\hline Urliste & praseznam \\
\hline verändern & spremeniti \\
\hline vermitteln & posredovati \\
\hline verweisen & usmerjati \\
\hline Verweisstruktur & kazalčna struktura \\
\hline verwenden & rabiti \\
\hline Verwendung & raba \\
\hline Verzeichnis & seznam \\
\hline Vorspann & uvodni del \\
\hline wissensbezogene Wörterbuchfunktion & slovarska funkcija v zvezi z znanjem \\
\hline Wörterbuchartikel & slovarski članek \\
\hline Wörterbuchbenutzungsforschung & raziskovanje rabe slovarja \\
\hline Wörterbuchbenutzungstest & test rabe slovarja \\
\hline Wörterbuchfunktion & funkcija slovarja \\
\hline Wörterbuchtyp & vrsta slovarja \\
\hline
\end{tabular}




\begin{tabular}{|l|l|} 
zergliedern & razčleniti \\
\hline zugreifen & dostopati \\
\hline Zugriff & dostop \\
\hline Zugriffsstruktur & struktura dostopanja \\
\hline
\end{tabular}

Tabelle 1: Lexikographische Termini und die auf dem Fachgebiet der Lexikographie verwendeten Termini/Lexeme im Deutschen mit den entsprechenden Äquivalenten im Slowenischen

\section{Anmerkungen}

1. Poethe führt an, dass der Grad der Normierung in den Geisteswissenschaften wesentlich niedriger ist als dies in den Naturwissenschaften der Fall ist (Poethe 2000: 202).

2. Terminologische Schulen unterschieden sich nach unterschiedlichen Ansätzen in der Praxis und unterschiedlichen Forschungsinteressen (vgl. Laurén und Picht 2006: 167).

3. Interessanterweise sind einige lexikographische Termini, darunter z. B. Lemmazeichen und Lemmazeichengestaltangabe im Slowenischen nicht belegt. Ins Deutsche wurden sie von Wiegand (1983) eingeführt.

4. $\quad$ Einige Bemerkungen zum Gebrauch von Daten und Informationen bei Wiegand (1998: 160-171).

5. Darunter vor allem die Fachpublikationen Slavistična revija und Jezik in slovstvo und die Monographie Terminologija in sodobna terminografija (2009). Seit 2013 erscheint die Fachpublikation Slovenščina 2.0.

6. Lehrbücher für den universitären Bereich, wie z. B. Lexikographie und Wörterbuchbenutzung (Engelberg und Lemnitzer 2009) und A Handbook of Lexicography: The Theory and Practice of Dictionary-making (Svensén 2009) gibt es im Slowenischen nicht.

7. Dies gelang mit dem Wörterbuch zur Lexikographie und Wörterbuchforschung (2010), womit ein wichtiger Beitrag im Sinne der Sammlung und Bearbeitung von lexikographischen Termini im Deutschen und Äquivalenten in neun Sprachen geleistet wurde und das Resultat internationaler Zusammenarbeit ist.

8. Mehr über die Tätigkeit des Instituts unter: http://isjfr.zrc-sazu.si/\#v [letzter Zugriff: 1. 8. 2016].

9. Mehr über die Tätigkeit des Instituts unter: http://www.ijs.si/ijsw/E8 [letzter Zugriff: 1. 8. 2016].

10. Mehr zur Tätigkeit von Trojina unter: http://www.trojina.si/ [letzter Zugriff: 1. 8. 2016].

11. Mehr über die Tätigkeit des Unternehmens unter: http://www.amebis.si/ [letzter Zugriff: 1. 8. 2016].

12. SP (2001) = Slovenski pravopis [Slowenisches Rechtschreibwörterbuch]

13. SSKJ2 (2014) = Slovar slovenskega knjižnega jezika 2 [Wörterbuch der slowenischen Standardsprache 2]

14. SSKJ (1994) = Slovar slovenskega knjižnega jezika [Wörterbuch der slowenischen Standardsprache]

15. Bei allen mit dem Asteriskus und Fragezeichen gekennzeichneten Belegen handelt es sich um terminologische Vorschläge. 
16. Viele der angeführten Ein- und Mehrwortbenennungen in der deutschen lexikographischen Terminologie gehen auf Wiegand zurück, der besonders für den Bereich der theoretischen Lexikographie viele beitrug.

17. Hier wird von der deutschen theoretischen Basis ausgegangen, deswegen ist die deutsche lexikographische Terminologie der Ausgangspunkt für die Bildung der fehlenden lexikographischen Terminologie im Slowenischen. Ohne eine theoretische Grundlage ist keine systematische Terminologie möglich.

18. Bei entlehnten Termini kann der Fall auftreten, dass Fachwörter, die den ursprünglichen lateinischen oder griechischen Terminus ersetzen, als Doubletten bestehen bleiben.

19. Der Terminus sestavljanje geht auf Toporišič zurück (2000: 159-160) und sestava auf Vidovič Muha (2011: 333)

20. Für die Zirkumfixderivation mit Substantiven konnten keine Belege gefunden werden.

21. Eichinger (2000: 73) zählt zu der morphologischen Konversion auch deverbale Konversionen, die mit einem Wechsel des Stammvokals verbunden sind, z. B. binden $\rightarrow$ Band.

22. Das Kurzwort OPAL ist 1. Objektbezogene Preis-, Aufwands- und Leistungsermittlung oder 2. Online publizierte Arbeiten zur Linguistik am IDS Mannheim. Das Kurzwort wird also auf zwei unverwandten Fachgebieten verwendet, wo es Unterschiedliches benennt.

23. Manchmal handelt es sich bei den Namen von Wörterbüchern, Datenbanken und anderen lexikographischen Produkten um Eponyme, vgl. z. B. Duden, Wahrig im Deutschen und Fran im Slowenischen.

24. Diese Formen sind leicht aussprechbar und wirken interessant. Bei solchen Formen handelt es sich oft um Produktnamen.

25. Dieses Wörterbuch enthält neue, noch nicht lexikalisierte Wörter.

26. Lehnwortbildung auf der Basis der gräkolateinischen Wortelemente erweist sich als vorteilhaft, da es vor allem in den Naturwissenschaften bereits eine gängige Praxis ist, sprachliches Material aus den klassischen Sprachen zu entlehnen (vgl. z. B. die botanischen Termini). Hier sei zudem bemerkt, dass die Begriffe 'klassisch' und 'modern' für die Bezeichnung von gräkolateinischen und englischen Elementen eher ungeschickt und falsch ist (Eins 2008: 4). Eins verweist nämlich darauf, dass sich die gräkolateinischen Elemente auch anderer Wortbildungsmuster als nur 'klassischen' bedienen können und die englischen Elemente auch zur Verbreitung der gräkolateinischen Konstituenten beigetragen haben (ebd.).

27. Vgl. auch oben unter Wortbildung.

28. Alle Beispiele stammen aus dem Glossar des Nachschlagewerks Computerlexikographie (Kunze und Lemnitzer 2007).

29. Als Quelle für die lexikographischen Termini im Deutschen dienten das Wörterbuch zur Lexikographie und Wörterbuchforschung (2010), Lexikographie und Wörterbuchbenutzung (Engelberg und Lemnitzer 2009), Computerlexikographie (Kunze und Lemnitzer 2007) und die Fachpublikation Lexicographica. Als Quelle für die slowenischen Äquivalente zu den deutschen lexikographischen Termini dienten die Beiträge der Fachpublikationen Slavistična revija, Jezik in slovstvo und die Außentexte der slowenischen Wörterbücher (zugänglich über das Portal Fran: http://www.fran.si/ [letzter Zugriff: 1. 8. 2016]). Bei einigen Äquivalenten im Slowenischen handelt es sich um Neubildungen. 


\section{Literatur}

Arntz, Reiner, Heribert Picht und Felix Mayer. 2009. Einführung in die Terminologiearbeit. 6. Aufl. Hildesheim/Zürich/New York: Georg Olms.

Bußmann, Hadumod. 1990. Lexikon der Sprachwissenschaft. Stuttgart: Alfred Kröner.

Butina-Koller, Ekaterina et al. 2010. Vorwort der Mitarbeiter zur terminologischen Äquivalenz. Wiegand, Herbert Ernst et al. (Hrsg.). 2010. Wörterbuch zur Lexikographie und Wörterbuchforschung (Band $1 \mathrm{~A}-\mathrm{C}$ ): XXV-XXX. Berlin/New York: Walter de Gruyter.

Cabré, Maria Teresa. 1999. Terminology. Theory, Methods and Applications. Amsterdam/Philadelphia: John Benjamins.

Donalies, Elke. 2002. Die Wortbildung des Deutschen. Ein Überblick. Tübingen: Gunter Narr.

Eichinger, Ludwig M. 2000. Deutsche Wortbildung. Eine Einführung. Tübingen: Narr.

Eins, Wieland. 2008. Muster und Konstituenten der Lehnwortbildung: Das Konfix-Konzept und seine Grenzen. Hildesheim: Georg Olms.

Engelberg, Stefan und Lothar Lemnitzer. 2009. Lexikographie und Wörterbuchbenutzung. 4. Aufl. Tübingen: Stauffenburg.

Fleischer, Wolfgang und Irmhild Barz. 1995. Wortbildung der deutschen Gegenwartssprache. 2. Aufl. Tübingen: Max Niemeyer.

Fleischer, Wolfgang und Irmhild Barz. 2012. Wortbildung der deutschen Gegenwartssprache. 4. Aufl. Berlin/Boston: Walter de Gruyter.

Fran. Slovarji Inštituta za slovenski jezik Frana Ramovša ZRC SAZU. http://www.fran.si/ [letzter Zugriff: 1. 8. 2016].

Jesenšek, Vida. 2013. Einführung. Fachsprachenlexikographie und Terminographie: eine kritischanalytische Betrachtung der Theorie und Praxis. Jesenšek, Vida (Hrsg.). 2013. Specialised Lexicography: 11-20. Berlin/Boston: Walter de Gruyter.

Jezik in slovstvo. http: //www.jezikinslovstvo.com [letzter Zugriff: 1. 8. 2016].

Kunze, Claudia und Lothar Lemnitzer. 2007. Computerlexikographie — Eine Einführung. Tübingen: Gunter Narr.

Laurén, Christer und Picht, Heribert. 2006. Approaches to Terminological Theories: A Comparative Study of the State-of-the-Art. Picht, Heribert (Hrsg.). 2006. Modern Approaches to Terminological Theories and Applications: 163-184. Bern/Berlin u. a.: Peter Lang.

Ledinek, Nina et al. (Hrsg.). 2009. Terminologija in sodobna terminografija. Ljubljana: Založba ZRC, ZRC SAZU.

Poethe, Hannelore. 2000. Fachsprachliche Aspekte der Wortbildung. Die Leistung der Wortbildung für Fachsprache und Fachtext. Barz, Irmhild, Marianne Schröder und Ulla Fix (Hrsg.). 2000. Praxis- und Integrationsfelder der Wortbildungsforschung: 199-218. Heidelberg: Winter.

Slavistična revija. http: //www.srl.si/ [letzter Zugriff: 1. 8. 2016].

Slovar slovenskega knjižnega jezika. 1994. Ljubljana: SAZU, ZRC SAZU in Inštitut za slovenski jezik Frana Ramovša.

Slovar slovenskega knjižnega jezika 2. 2014. SAZU, ZRC SAZU in Inštitut za slovenski jezik Frana Ramovša.

Slovenski pravopis. 2001. Ljubljana: SAZU, ZRC SAZU in Inštitut za slovenski jezik Frana Ramovša. 
Svensén, Bo. 2009. A Handbook of Lexicography. The Theory and Practice of Dictionary-Making. Cambridge u. a.: Cambridge University Press.

Toporišič, Jože. 2000. Slovenska slovnica. 4. Aufl. Maribor: Obzorja.

Vidovič Muha, Ada. 2011. Slovensko skladenjsko besedotvorje. Ljubljana: Znanstvena založba Filozofske fakultete Univerze v Ljubljani.

Vintar, Špela. 2008. Terminološka veda in računalniško podprta terminografija. Ljubljana: Filozofska fakulteta.

Wiegand, Herbert Ernst. 1983. Was ist eigentlich ein Lemma? Ein Beitrag zur Theorie der lexikographischen Sprachbeschreibung. Wiegand, Herbert Ernst (Hrsg.). 1983. Studien zur neuhochdeutschen Lexikographie III: 401-474. Germanistische Linguistik 1-4/82. Hildesheim u. a.: Georg Olms Verlag.

Wiegand, Herbert Ernst. 1998. Wörterbuchforschung. Untersuchungen zur Wörterbuchbenutzung, zur Theorie, Geschichte, Kritik und Automatisierung der Lexikographie. 1. Teilband. Mit 159 Illustrationen im Text. Berlin/New York: Walter de Gruyter.

Wiegand, Herbert Ernst et al. (Hrsg.). 2010. Wörterbuch zur Lexikographie und Wörterbuchforschung. (Band 1 A-C). Berlin/New York: Walter de Gruyter. 